\title{
Intravesical formalin for hemorrhagic cystitis: A contemporary cohort
}

\author{
Matthew J. Ziegelmann, MD; Stephen A. Boorjian, MD; Daniel D. Joyce, BSc; Brian D. Montgomery, MD; \\ Brian J. Linder, MD
}

Department of Urology, Mayo Clinic, Rochester, MN, United States

Cite as: Can Urol Assoc J 2017;11 (3-4):E79-82. http://dx.doi.org/10.5489/cuaj.4047

Published online March 16, 2017

\section{Abstract}

Introduction: Hemorrhagic cystitis presents a difficult clinical challenge, yet data regarding treatment options is sparse. Here, we sought to evaluate outcomes of a contemporary cohort of patients treated with intravesical formalin for hemorrhagic cystitis.

Methods: We identified a retrospective cohort of eight patients managed with formalin for hemorrhagic cystitis from 2000-2014. All patients failed prior measures, including bladder irrigation, clot evacuation, and other intravesical agents. Treatment success was defined as hematuria resolution during the given hospitalization without use of additional invasive therapies. We also evaluated treatment complications and additional treatments following hospital dismissal.

Results: Etiology of cystitis was radiation for malignancy in all cases. The formalin concentration ranged from $1-4 \%$, with escalation used in treatment failures. Five patients $(62.5 \%)$ received a single dose of $1 \%$ formalin, two patients received two doses, and one patient received three doses. Notably, intraoperative cystography identified vesicoureteral reflux (VUR) in $50.0 \%$ of patients. Six patients $(75.0 \%)$ achieved treatment success, with a median time to resolution of four days (range 1-17 days). Of those refractory to formalin, one was managed with indwelling nephrostomy tubes and one underwent cystectomy. Median followup was eight months. Of the responders, two eventually required cystectomy, one for recurrent hematuria and one for recalcitrant bladder neck contracture and bladder dysfunction. The remaining four patients (50\%) required no additional therapy.

Conclusions: Formalin remains an important tool for treating refractory hemorrhagic cystitis, with roughly $75.0 \%$ of patients requiring no additional therapy prior to hospital discharge. Notably, there is a risk of bladder dysfunction following formalin.

\section{Introduction}

Hematuria accounts for almost $30 \%$ of all urological evaluations and can be caused by a multitude of benign and malignant processes. ${ }^{1}$ One such entity is hemorrhagic cys- titis, which is characterized by diffuse bleeding throughout the urothelium and is typically secondary to radiation or chemotherapy exposure..$^{-5}$ Notably, the presentation of hemorrhagic cystitis may vary from mild, self-limited gross hematuria to refractory, life-threatening hemorrhage with clot retention requiring blood transfusion and emergent surgical or radiological intervention. ${ }^{6}$

Unfortunately, despite the clinical challenge that severe hemorrhagic cystitis can present, data regarding treatment options is sparse. Notwithstanding this inherent limitation, several authors have proposed treatment algorithms for hemorrhagic cystitis. ${ }^{2,7}$ These typically progress from less invasive options, such as bladder irrigation or fulguration, to more aggressive measures, such as internal iliac angioembolization or even cystectomy with urinary diversion for refractory cases. Notably, while commonly used, there is little supporting literature to define outcomes with intravesical therapies, such as alum, silver nitrate, and formalin. ${ }^{2,5}$

Intravesical formalin instillation, first reported in 1969, was initially used in the management of refractory bladder hemorrhage from inoperable cancer. ${ }^{8}$ The proposed mechanism of action involves capillary occlusion and protein fixation at the level of the urothelium. ${ }^{9,10}$ Since the initial report, multiple series have suggested a role for intravesical formalin in the treatment of refractory bladder hemorrhage, with reported success rates ranging from $70-90 \%$, with variations in concentrations instilled and definitions of success. ${ }^{9,11,12}$ Additionally, these reports are typically small series with limited followup, and represent historical cohorts. Therefore, we evaluated the clinical presentation, management, and outcomes of intravesical formalin in the setting of hemorrhagic cystitis in a contemporary cohort of patients.

\section{Methods}

Following Institutional Review Board approval, we identified 104 patients who were hospitalized for hemorrhagic cystitis between 2004 and 2014. Of those, eight were treated with intravesical formalin during the index hospitalization. All 
patients were 18 years or older at the time of treatment and had failed initial measures, including indwelling catheter $(n=8)$, continuous normal saline bladder irrigation $(n=8)$, and intravesical therapy with agents such as alum $(n=6)$, silver nitrate $(n=4)$, and aminocaproic acid $(n=2)$.

Intravesical formalin instillation was performed under a general anesthesia in all cases. Rigid cystoscopy was performed first for clot evacuation and fulguration of any discrete bleeding vessels. A large bore catheter was then placed and a cystogram was performed with instillation of $300 \mathrm{cc}$ of contrast into the bladder to evaluate for bladder perforation, as well as evidence of vesicoureteral reflux (VUR). If reflux was identified, Fogarty catheters were used to occlude the ureteral orifice(s) in an attempt to prevent formalin reflux into the upper tract. The external genitalia was protected from formalin exposure. For men, this was performed by carefully wrapping the penis with plastic wrap after applying copious amounts of petroleum jelly to the area. Similar skin precautions were performed in females. After this, any exposed areas (e.g., penis, perineum, and suprapubic areas) were then covered with sterile surgical towels. In addition, the indwelling catheter balloon was placed on slight tension against the bladder neck to prevent urethral exposure. Next, the formalin solution (range 1-4\%) was instilled into the bladder under gravity at $<15 \mathrm{~cm}$ of pressure with a dwell time of 10-15 minutes. After draining the formalin from the bladder, the bladder was copiously irrigated with sterile water and continuous bladder irrigation with normal saline was started.

Patient charts were reviewed for clinicopathological variables, including age at the time of formalin administration, etiology for hemorrhagic cystitis, medical comorbidities, previous therapies for hemorrhagic cystitis, perioperative complications (within 30 days), blood transfusion after formalin installation, hematuria resolution, and overall survival. The Clavien-Dindo system was used to grade post-treatment complications.$^{13}$ The retrospective nature of this study precluded a standardized followup protocol, and patient followup after hospitalization was directed by the treating physicians. Treatment success was defined as resolution of hematuria during the given hospitalization without use of additional therapies other than normal saline bladder irrigation.

\section{Results}

Of the eight patients who underwent intravesical formalin treatment for hemorrhagic cystitis between 2000 and 2014, six were male $(75 \%)$ and two were female $(25 \%)$. Clinical and demographic variables are shown in Table 1. Median age at the time of presentation was 83 years (interquartile range [IQR] 79.6-83.5), and in all cases hemorrhagic cystitis was secondary to external beam radiation therapy. Radiation therapy was administered for prostatic adenocarcinoma in the six males and cervical malignancy in the two females.
The median time from radiation treatment to intravesical formalin instillation was 98 months (IQR 71-126).

All patients were hospitalized at the time of formalin instillation. Of note, all eight patients had previously been treated with cystoscopy, clot evacuation, and normal saline bladder irrigation. Additionally, seven patients (88\%) were treated with prior intravesical therapy, including alum in six $(75 \%)$, silver nitrate in four $(50 \%)$, and aminocaproic acid in two (25\%). Additionally, two patients (25\%) were treated with hyperbaric oxygen therapy (Table 2). Formalin instillation initially consisted of a $1 \%$ solution in all patients. Notably, three patients underwent a second instillation of formalin (1-2\% formalin), and one patient underwent a third treatment (4\% formalin). Four patients $(50 \%)$ had evidence of reflux on cystogram, and Fogarty catheters were used to provide ureteral orifice occlusion with verification on repeat cystogram prior to formalin instillation (Table 3). Importantly, new onset VUR was identified in one patient at the time of the second formalin instillation.

There were no intraoperative complications. Formalin treatment was successful in six patients $(75 \%)$, with resolution of hematuria during the given hospitalization without additional inpatient treatment at a median of four days post-installation (range 1-17 days). Notably, three patients (38\%) experienced immediate resolution. Severe complications occurred in one patient. This was a Clavien IVa complication, including postoperative acute kidney injury (non-obstructive) and respiratory distress requiring a stay in the intensive care unit. Five patients $(63 \%)$ required blood transfusion within 30 days of formalin instillation, classified as Clavien II complication. The median hospital duration was 31 days (range 2-45 days), including four patients $(50 \%)$ with hospital stays greater than 30 days. The median length of stay after formalin instillation was 12 days (range 2-113 days) for the total cohort. Of the six responders, the

\section{Table 1. Patient clinical and demographic features}

\begin{tabular}{lc}
\hline Feature & $\begin{array}{c}\text { Intravesical formalin } \\
\text { instillation (N=8) }\end{array}$ \\
\hline Median age (yrs), median, (IQR) & $83(79.5-83.5)$ \\
Gender, $\mathrm{n}(\%)$ & $6(75)$ \\
Male & $2(25)$ \\
Female & \\
Hemorrhagic cystitis etiology, n (\%) & $6(75)$ \\
XRT for PCa & $2(25)$ \\
XRT for GYN Ca & $30.2(23.5-31.8)$ \\
Median BMI, n (IQR) & $1(13)$ \\
Diabetes mellitus, n (\%) & $5(63)$ \\
Hypertension, n (\%) & $2(25)$ \\
Coronary artery disease, n (\%) & $2(25)$ \\
Current or previous smoker, $n$ (\%) & \\
\hline BMl: body mass index; IQR: interquartile range; GYN Ca: gynecological cancer; \\
PCa: prostate cancer; XRT: external beam radiation.
\end{tabular}




\begin{tabular}{lc}
\hline Table 2. Prior hemorrhagic cystitis treatment \\
\hline Treatment & $\begin{array}{c}\text { Intravesical formalin } \\
\text { instillation (N=8) }\end{array}$ \\
\hline Continuous bladder irrigation, $\mathrm{n}(\%)$ & $8(100)$ \\
Cystoscopy + clot evacuation, $\mathrm{n}(\%)$ & $8(100)$ \\
Intravesical alum, $\mathrm{n}(\%)$ & $6(75)$ \\
Silver nitrate, $\mathrm{n}(\%)$ & $4(50)$ \\
Aminocaproic acid, $\mathrm{n}(\%)$ & $2(25)$ \\
Hyperbaric oxygen therapy, $\mathrm{n}(\%)$ & $2(25)$ \\
\hline
\end{tabular}

median hospital stay after formalin instillation was nine days (range $2-17$ days).

Only one of the six patients (17\%) who initially responded to formalin instillation had a recurrence of hematuria and underwent planned cystectomy. Also, of the two patients who did not respond to formalin, one underwent cystectomy with ileal conduit urinary diversion during the same hospitalization, while the second patient was managed with bilateral indwelling percutaneous nephrostomy tube drainage due to the patient's complex medical comorbidities and poor functional status, precluding cystectomy. Finally, the single patient who was treated with formalin dose escalation ultimately developed a severely contracted bladder with refractory urinary urgency, urge incontinence, and recurrent bladder neck contracture. This was initially managed with cystoscopic bladder neck incision and anticholinergic therapy. However, this patient ultimately underwent delayed planned cystectomy for refractory symptoms approximately eight months after formalin instillation.

The median followup for our cohort was eight months (range 3-72). During this time, four patients died unrelated to their hemorrhagic cystitis. The one-year overall survival rate following formalin instillation was $75 \%$ and the threeyear overall survival rate was $50 \%$.

\section{Discussion}

In our series of patients undergoing intravesical formalin therapy for the treatment of refractory hemorrhagic cystitis, we found that $75 \%$ experienced hematuria resolution during the immediate hospitalization. Additionally, $50 \%$ of patients had a durable resolution of hematuria at a median of eight months. Our data augments the current literature by evaluating a contemporary cohort of patients with intermediateterm followup. Further, recognition that one patient (13\%) underwent simple cystectomy for refractory voiding dysfunction may aid clinicians in discussing and evaluating the risks of formalin instillation during preoperative decision-making.

Intravesical formalin, a concentrated formaldehyde solution, has long been used in the medical field as an antiseptic, disinfectant, tissue fixative, and embalming solution. ${ }^{12}$ Formalin works by inducing protein fixation at the level of the capillaries. ${ }^{14}$ Notably, it was not until 1969 that Brown

\begin{tabular}{lc}
\hline Table 3. Perioperative and postoperative outcomes \\
\hline Outcome & $\begin{array}{c}\text { Intravesical formalin } \\
\text { instillation (N=8) }\end{array}$ \\
\hline Total formalin instillations, $\mathrm{n}(\%)$ & $5(63)$ \\
1 & $2(25)$ \\
2 & $1(13)$ \\
3 & $10(10-15$ mins) \\
Median dwell time in mins (range) & $4(50)$ \\
Reflux, $\mathrm{n}(\%)$ & \\
30 -day complications, $\mathrm{n}(\%)$ & $0(0)$ \\
Clavien I & $5(63)$ \\
Clavien II & $0(0)$ \\
Clavien III & $1(13)$ \\
Clavien IV & $6(75)$ \\
Resolution of hematuria, $\mathrm{n}(\%)$ & $4(1-17$ days) \\
Median time to resolution in days (range) & $4(50)$ \\
Additional procedures, $\mathrm{n}(\%)$ & $1(13)$ \\
Bilateral nephrostomy tube placement & $3(38)$ \\
Cystectomy with urinary diversion &
\end{tabular}

first described the use of intravesical formalin for the treatment of refractory hemorrhage in a cohort of 24 patients with advanced bladder malignancy. ${ }^{8} \mathrm{He}$ found that $90 \%$ of patients experienced an improvement in their hematuria for an average of four months after instillation of $10 \%$ formalin into the bladder.

More recently, intravesical formalin success rates have been reported from 70-90\%. ${ }^{9,11,12,15-17}$ In their comprehensive review of the 235 previously reported cases of formalin use, Donahue and Frank (1989) identified a trend towards increasing complete response rates with $10 \%$ intravesical formalin (83\% complete response), as compared to $5 \%$ formalin (78\% complete response) or $1 \%$ formalin ( $71 \%$ complete response) solutions, although this was not statistically significant. ${ }^{16}$ Here, we identified a success rate of $63 \%$ at a median followup of eight months with the initial use of $1 \%$ formalin. Notably, dose escalation to $4 \%$ was used in only one patient. Differences in our success rate compared to those previous reported may be secondary to disparate patient populations, differences in the definition of success, availability of other contemporary treatments, and formalin concentrations.

Risks associated with formalin instillation, such as hydroureteronephrosis, acute renal injury, reduced bladder capacity, and urinary tract fistulae, have been described. ${ }^{12,16,18}$ From the limited available literature, it is clear that higher formalin concentrations are associated with an increased risk for adverse events. ${ }^{12,16}$ Serious complication rates, as high as $50-75 \%$, have been reported in patients undergoing 10\% formalin instillation, albeit in small series. ${ }^{11,15}$ For instance, in one series, $40 \%$ of patients required urinary diversion after intravesical formalin instillation for complications related to the procedure..$^{18}$ In contrast, we report that one of the eight patients $(13 \%)$ underwent simple cystectomy secondary to 
bladder damage from formalin instillation. Interestingly, this was the only patient in our cohort treated with dose escalation. Additionally, one patient developed new onset, unilateral VUR during treatment. This patient had an initial cystogram performed, which was without evidence of reflux. However, the patient was taken back for additional formalin therapy, and at that time, repeat cystogram showed unilateral reflux. One hypothesis is that the formalin therapy induced bladder dysfunction by altering the anti-reflux mechanism of the vesicoureteral junction. Alternatively, the reflux may have been missed with the initial cystography. These results highlight the importance of evaluating for reflux via cystogram prior to each formalin instillation and the potential risk of severe bladder dysfunction following treatment.

Despite a greater than $60 \%$ success rate with intravesical formalin therapy in our cohort, three patients did require additional therapy for refractory hemorrhage, including two patients $(25 \%)$ who underwent cystectomy and one patient $(13 \%)$ who was managed with bilateral nephrostomy tubes for permanent urinary diversion. Given the risk-benefit profile of formalin instillation, we advocate its potential role in the management of hemorrhagic cystitis following failure of other intravesical agents. However, given the high rates of complications and even postoperative mortality reported in patients requiring cystectomy for hemorrhagic cystitis, use of formalin prior to surgical extirpation may be prudent. ${ }^{2,6}$

Limitations of our series should be noted, including the small cohort size and retrospective design. Notably, multiple surgeons were involved in the care of these patients over a 14-year time span and as such, the decision to proceed with intravesical formalin was not standardized. Additionally, given the nature of our practice as a tertiary referral centre, we did not have a standardized followup; thus, patients may have sought additional therapy or received emergent therapy at other institutions.

\section{Conclusion}

Although there is a risk for significant bladder dysfunction, especially with greater formalin concentrations, $75 \%$ of our patients experienced resolution of their hematuria with intravesical formalin therapy. This therapy remains an important treatment modality for patients with refractory hemorrhagic cystitis prior to more invasive therapies, such as internal iliac artery angioembolization or urinary diversion.
Competing interests: The authors report no competing personal or financial interests.

This paper has been peer-reviewed.

\section{References}

1. Antoniewicz AA, Zapała L, Poletajew S, et al. Macroscopic hematuria-a leading urological problem in patients on anticoagulant therapy: Is the common diagnostic standard still advisable? ISRN Urology 2012; 2012: 710734 .

2. Linder BJ, Boorijan SA. Management of emergency bleeding, recalcitrant clots, and hemorrhagic cystitis. AUA Update Series 2015;34(Lesson 3).

3. Lojanapiwat B, Sripralakrit S, Soonthornphan $S$, et al. Intraveside formalin instillation with a modified technique for controlling hemorrhage secondary to radiation cystitis. Asian J Surg 2002;25: 232-5. https://doi.org/10.1016/S1015-9584(09)60181-0

4. Corman JM, McClure $D$, Pritchett $R$, et al. Treatment of radiation induced hemorrhagic cystitis with hyperbaric oxygen. J Urol 2003;169: 2200-2. https://doi.org/10.1097/01.ju.0000063640.41307.c9

5. Payne H, Adamson A, Bahl A, et al. Chemical- and radiation-induced haemorrhagic cystitis: Current treatments and challenges. BJU Int 2013;112:885-97. https://doi.org/10.1111/bju.12291

6. Linder BJ, Tarrell RF, Boorijan SA. Cystectomy for refractory hemorrhagic cystitis: Contemporary etiology, presentation and outcomes. J Urol 2014;192:1687-92. https://doi.org/10.1016/i.juro.2014.06.030

7. Rastinehad AR, Kavoussi LR, Noble MJ. Hemorrhagic cystitis. AUA Update Series 2007;26(Lesson 7).

8. Brown RB. A method of management of inoperable carcinoma of the bladder. Med J Aust 1969;1:23-4.

9. Shah $B C$, Albert DJ. Intravesical instillation of formalin for the management of intractable hematuria. J Urol 1973;1 10:519-20.

10. Choong SK, Walkden M, Kirby R. The management of intractable haematuria. BJU Int 2000; 86:951-9. https://doi.org/10.1046/i.1464-410x.2000.00900.x

11. Fair WR. Formalin in the treatment of massive bladder hemorrhage. Techniques, results, and complications. Urology 1974;3: 573-6. https://doi.org/10.1016/S0090-4295(74)80250-5

12. Godec CJ, Gleich P. Intractable hematuria and formalin. J Urol 1983;130: 688-91.

13. Dindo D, Demartines N, Clavien PA. Classification of surgical complications: A new proposal with evaluation in a cohort of 6336 patients and results of a survey. Ann Surg 2004;240:205-13. https://doi.org/10.1097/01.sla.0000133083.54934.ae

14. Bergreen PW, Ayala AG, Johnson DE. Effect of topical formaldehyde on canine bladder. Urology 1976;7:279-82. https://doi.org/10.1016/0090-4295(76)90458-1

15. Vicente J, Rios $G$, Caffaratti J. Intravesical formalin for the treatment of massive hemorrhagic cystitis: Retrospective review of 25 cases. Eur Urol 1990; 18:204-6.

16. Donahue LA, Frank IN. Intravesical formalin for hemorrhagic cystitis: Analysis of therapy. J Urol 1989;141: 809-12.

17. Giannakopoulos $X$, Grammeniatis $E$, Chambilomatis $P$, et al. Massive hemorrhage of inoperable bladder carcinomas: Treatment by intravesical formalin solution. Int Urol Nephrol 1997;29: 33-8. https://doi.org/10.1007/BF02551414

18. Fall M, Pettersson S. Ureteral complications after intravesical formalin instillation. J Urol 1979;122:160-2.

Correspondence: Dr. Brian J. Linder, Department of Urology, Mayo Clinic, Rochester, MN, United States; Linder.Brian@mayo.edu 